\title{
Erratum: Observation of an evolving magnetic flux rope before and during a solar eruption
}

Jie Zhang, Xin Cheng \& Ming-de Ding

Nature Communications 3:747 doi: 10.1038/ncomms1753 (2012); Published 20 Mar 2012; Updated 7 Jun 2016

The HTML version of this Article previously published omitted Supplementary Movie 2. This has now been corrected in the HTML version of the Article; the PDF was correct from the time of publication. 